\title{
EPIDEMIOLOGIA DO ESGOTO COMO ESTRATÉGIA PARA MONITORAMENTO COMUNITÁRIO, MAPEAMENTO DE FOCOS EMERGENTES E ELABORAÇÃO DE SISTEMAS DE ALERTA RÁPIDO PARA COVID-19
}

\author{
Fernando F. Sodréa, $*,\left(\mathbb{D}\right.$, Cristina C. S. Brandão ${ }^{\mathrm{b}}$, Carla S. Vizzotto ${ }^{\mathrm{b}}$ e Adriano O. Maldaner \\ Instituto de Química, Universidade de Brasília, 70910-000 Brasília - DF, Brasil \\ bDepartamento de Engenharia Civil e Ambiental, Universidade de Brasília, 70910-000 Brasília - DF, Brasil \\ 'Instituto Nacional de Criminalística, Polícia Federal, SAIS Quadra 07 Lote 23, 70610-200 Brasília - DF, Brasil,
}

Recebido em 28/04/2020; aceito em 04/05/2020; publicado na web em 06/05/2020

\begin{abstract}
WASTEWATER-BASED EPIDEMIOLOGY AS A STRATEGY FOR COMMUNITY MONITORING, MAPPING OF HOTSPOTS AND EARLY WARNING SYSTEMS OF COVID-19. The ability to monitor the spread of diseases is essential for prevention, intervention and control practices. In this context, wastewater-based epidemiology (WBE) has been applied to empirically evaluate the exposure of populations to chemical substances and pathogens of emerging concern in near real time. During the current COVID-19 pandemic, WBE remains an innovative epidemiological tool, with the potential to complement infectious disease surveillance systems. Recent studies on WBE and COVID-19 are based on the monitoring of viral RNA fragments as target biomarkers for SARS-CoV-2 via RT-qPCR and allied techniques. Literature results have been shown the potential of WBE as an innovative tool to monitor viral spread in large communities, map contagion curves and hotspots, identify the contribution of asymptomatic individuals, and subsidize early warning systems for disease outbreaks. WBE can offer less expensive responses for monitoring large populations compared to individual clinical tests which are not feasible on large scales. This aspect is important in terms of health surveillance and associated public policies, notably where clinical tests are scarce or underestimated and in less developed regions with inadequate sanitation conditions.
\end{abstract}

Keywords: Wastewater-based epidemiology; COVID-19; SARS-CoV-2; novel coronavirus; human health risks.

\section{CONTEXTUALIZAÇÃO}

A quantificação de moléculas de drogas ilícitas, seus metabólitos e adulterantes em amostras de esgoto tem permitido estimar o consumo comunitário de cocaína, cannabis, anfetaminas, opioides, novas drogas sintéticas, dentre outras, de maneira empírica, não invasiva e em tempo quase-real. ${ }^{1,2}$ Essa estratégia, denominada epidemiologia do esgoto (do inglês, Wastewater-Based Epidemiology - WBE), tem complementado estimativas obtidas por métodos usuais ${ }^{3}$ e subsidiado os mais recentes relatórios do Observatório Europeu da Droga e da Toxicodependência ${ }^{4}$ e do Escritório das Nações Unidas sobre Drogas e Crime. ${ }^{5}$

No Brasil, estimativas do consumo de drogas de abuso via WBE, sob diferentes condições espaciais e temporais, têm sido produzidas há cerca de dez anos, especificamente no Distrito Federal (DF), por interveniência do Instituto Nacional de Ciências e Tecnologias Analíticas Avançadas (INCTAA) e com participação do Instituto de Química da UnB, do Instituto Nacional de Criminalística da Polícia Federal, do Departamento de Polícia Técnica da Polícia Civil do DF e da Companhia de Saneamento Ambiental do DF (CAESB). ${ }^{6}$ De forma ilustrativa, os resultados obtidos no DF apontam consistentemente para um aumento do consumo de cocaína nos finais de semana, que praticamente dobra em comparação aos outros dias indicando um incremento do uso recreativo da cocaína, ou seja, usuários esporádicos que consomem drogas apenas em eventos sociais ou festivos, ou ainda usuários compulsivos que aumentam o uso nestes dias.

A WBE aumentou o conhecimento recente sobre padrões de consumo de drogas e mostra potencial crescente para medir aspectos socioeconômicos e relacionados à saúde pública. ${ }^{7}$ Mais recentemente, esta estratégia tem sido ajustada para identificar a exposição de

*e-mail: ffsodre@unb.br populações a produtos químicos, ${ }^{8,9}$ pesticidas, ${ }^{10}$ micotoxinas, ${ }^{11}$ dietas alimentares ${ }^{9}$ e doenças infecciosas. ${ }^{12}$

\section{Monitoramento de doenças infecciosas via WBE}

A capacidade de monitorar rapidamente a propagação de doenças é essencial para prevenção, intervenção e controle. No entanto, existem limitações dos atuais sistemas de vigilância para lidar com o rápido crescimento populacional, com o surgimento de novos organismos patogênicos e com ressurgimento de infecções outrora controladas. Neste contexto, a WBE se coloca como uma nova ferramenta epidemiológica, com potencial para complementar sistemas de vigilância de doenças infecciosas e subsidiar sistemas de alerta precoce para surtos de doenças. Suas vantagens frente a outras técnicas de vigilância em saúde pública são a capacidade de monitorar tendências espaciais e temporais, produzir resultados em tempo quase-real ou real (via biossensores) e gerar informações sobre toda uma população sem a necessidade de aprovação por comitês de ética, a depender do tamanho da região urbana estudada. ${ }^{13}$

Ao contrário dos protocolos amplamente estabelecidos e constantemente validados para estimar o consumo de drogas via WBE, o monitoramento de doenças infecciosas, por ser mais recentemente explorado, demanda ainda mais o esforço conjunto de diferentes áreas de conhecimento. Os principais desafios envolvem a seleção de biomarcadores estáveis nas águas residuárias, ${ }^{13,14}$ possíveis incertezas relacionadas à contribuição da população ${ }^{13,15} \mathrm{e}$ o intervalo de tempo entre a coleta e análise dos dados. ${ }^{13}$ Embora existam desafios porvir, é notável a mobilização recente da comunidade científica em torno do tema, haja vista a pandemia causada pelo novo coronavírus, o SARS-CoV-2, causador da COVID-19. A capacidade de determinar rapidamente o status e as tendências da COVID-19 dentro e entre as comunidades é o maior trunfo da WBE. ${ }^{14,15}$ Além disso, esta estratégia 
deve oferecer respostas de maneira menos onerosa, pois os testes de diagnóstico clínico da COVID-19 são inadequados, sob o ponto de vista financeiro, para monitorar rapidamente a incidência da doença em toda uma população. Ou seja, não é viável fazer testes individuais repetidos em escalas muito grandes. ${ }^{14}$

\section{WBE para monitoramento do novo coronavírus}

Os vírus apresentam baixa complexidade morfológica, consistindo em um material genético, um capsídeo e, em alguns casos, um envelope. Não possuem aparato enzimático suficiente para replicação, necessitando de células hospedeiras para completar o seu ciclo. ${ }^{16} \mathrm{~A}$ entrada do SARS-CoV-2 em células humanas é mediada por glicoproteínas (spike $-S$ protein) que se projetam da superfície viral e interagem fortemente com a enzima conversora de angiotensina 2 (ACE2). ${ }^{17,18}$ Humanos infectados podem disseminar partículas virais de SARS-CoV-2 que se mantem viáveis sob diferentes condições ambientais e em diferentes superfícies. ${ }^{19}$ Embora a transmissão direta de gotículas seja uma importante via de transmissão, a excreção fecal e a subsequente contaminação ambiental também podem levar à presença de partículas viáveis de SARS-CoV-2.20

O monitoramento de SARS-CoV-2 em amostras fecais e respiratórias de pacientes infectados pela COVID-19 foi feito recentemente por qRT-PCR (reverse transcription-quantitative polymerase chain reaction), uma técnica de transcrição reversa e reação em cadeia da polimerase amplamente utilizada para verificar a expressão gênica, ou seja, identificar e quantificar o RNA em amostras ${ }^{21}$. Pacientes com amostras fecais positivas (55\% do total) apresentaram resultados respiratórios que permaneceram positivos para o RNA do SARSCoV-2 por uma média de 16,7 dias, enquanto que nas amostras fecais os resultados permaneceram positivos por uma média de 27,9 dias após o início dos primeiros sintomas, ou seja, em média 11,2 dias a mais do que nas amostras respiratórias. ${ }^{21}$ Os autores sugeriram uma dispersão viral prolongada pelas fezes, por quase cinco semanas após as amostras respiratórias dos pacientes terem resultado negativo para RNA do SARS-CoV-2.

A carga de partículas virais no esgoto pode ser medida ao se buscar motivos funcionais ou estruturais do vírus. Mesmo que, segundo a Organização Mundial de Saúde, a meia-vida de partículas viáveis de SARS-CoV-2 em águas residuárias seja curta, ${ }^{22}$ provavelmente em função das condições adversas à manutenção de seu envelope viral lipídico, ainda não há estudos sobre a viabilidade de SARS-CoV-2 em esgotos. Mesmo assim, há diferentes estratégias para detecção de partículas viáveis e não-viáveis que podem ser empregadas para se identificar a presença do vírus. ${ }^{14}$ Em termos de biossegurança, a transmissão entérica do SARS-CoV-2 é possível e pode representar em risco para a saúde de trabalhadores em estações de tratamento, ${ }^{23}$ caso não sejam seguidos os protocolos de higiene preconizados pela $\mathrm{OMS}^{22}$ e comumente usados para proteção geral por qualquer doença infecciosa, incluindo a COVID-19. Também há preocupação com a possibilidade de transmissão fecal-oral da COVID-19, especialmente em áreas com falta de saneamento, onde a capacidade de diagnóstico pode ser limitada.

Produtos de degradação, como fragmentos de RNA, têm sido recentemente selecionados como biomarcadores-alvo. ${ }^{15}$ As abordagens de detecção podem incluir RT-PCR (e abordagens aliadas), mas também ELISA, juntamente com o método do número mais provável (MPN) para quantificar cargas de partículas combinadas de vírus viáveis e não-viáveis. ${ }^{14}$

Os primeiros resultados positivos para a presença de SARSCoV-2 em amostras de esgoto foram publicados na Holanda ao se investigar a presença de fragmentos do RNA viral por qRT-PCR em águas residuárias de sete estações de tratamento de esgotos (ETE) e de um aeroporto. ${ }^{24}$ Os resultados das amostras de 6 de fevereiro de 2020, três semanas antes do primeiro caso ser relatado na Holanda, não mostraram sinais positivos para os conjuntos de primers (oligonucleotídeos iniciadores) N1-3 (nucleocapsídeos) e E (envelope viral), conforme mostrados na Tabela 1. Amostras de 4 e 5 de março, uma semana após a confirmação da epidemia, com 38 e 82 casos oficiais da COVID-19, respectivamente, mostraram sinal positivo para o primer $\mathrm{N} 1 \mathrm{em}$ amostras de quatro das ETEs investigadas. ${ }^{24}$ Nove dias depois (15 e 16 de março), seis das sete ETEs apresentaram sinal positivo para N1, cinco ETEs para N3 e quatro ETEs para o primer E. Segundo os autores, os resultados encontrados corresponderam parcialmente com a sensibilidade observada em análises clínicas de SARS-CoV-2, onde $\mathrm{N} 1=\mathrm{N} 3>\mathrm{N} 2$, evidenciando a capacidade inequívoca do uso de águas residuárias para monitoramento da dispersão viral, bem como para a criação de um sistema de vigilância em saúde baseado na análise de esgotos.

Alguns dias depois dos resultados disponibilizados na Holanda, foram detectados altos títulos de SARS-CoV-2, por qRT-PCR e sequenciamento direto de DNA, em águas residuárias coletadas entre 18 e 25 de março em uma grande ETE de Massachusetts, nos Estados Unidos. ${ }^{25}$ Os títulos virais observados foram significativamente mais altos do que o esperado em comparação aos casos clinicamente confirmados em Massachusetts a partir de 25 de março. ${ }^{25}$ Embora os autores não tenham encontrado um motivo para esta discrepância, observaram que os resultados foram consistentes com a ideia de que uma fração significativa de casos não é detectada pelos atuais algoritmos de teste e que essa fração poderia incluir um grande número de pacientes assintomáticos. Ainda, sugeriram que áreas de diferentes dimensões, dentro de uma mesma região geográfica, devem ter suas águas residuárias investigadas para que seja possível estimar a prevalência da doença independentemente de se conhecer o título viral médio nas fezes infectadas. ${ }^{25}$

Além dos resultados pioneiros obtidos na Holanda e nos Estados Unidos, a revista Nature noticiou no início de abril que vários grupos de pesquisa já iniciaram análises de águas residuárias para investigar a presença do novo coronavírus. ${ }^{15}$ Segundo a notícia, resultados positivos já haviam sido encontrados em amostras coletadas na Suécia, além da Holanda e dos Estados Unidos. Existe também uma mobilização crescente de diferentes grupos de pesquisa de todo mundo que estão se alinhando em busca do compartilhamento de protocolos e experiências. ${ }^{26,27}$

Mais recentemente, entre os dias 5 e 8 de abril de 2020, 23 amostras de esgoto bruto e oito de esgoto tratado foram investigadas para o primer E do SARS-CoV-2 em três das principais ETEs de Paris. ${ }^{28}$ Os autores, observaram resultado positivo para todas as amostras brutas, além de resultados positivos em seis das oito amostras de efluentes das ETE, com uma redução da carga viral de 100 vezes. Os autores mostraram que o aumento das unidades de SARS-CoV-2 nas amostras acompanhou, com precisão, a evolução do número de casos fatais observados, tanto em Paris quanto na França. Finalmente, os autores alertaram para o fato de que a contaminação das águas residuárias ocorreu antes do crescimento exponencial do número de casos, evidenciando a capacidade do monitoramento sistemático de esgotos como uma estratégia alternativa e rápida para detectar patógenos em populações, notadamente em situações nas quais investigações clínicas em indivíduos tornam-se difíceis por razões logísticas, éticas ou econômicas. ${ }^{28}$

Há notícias sinalizando que o monitoramento de águas residuárias está ocorrendo também em outros países como Austrália, ${ }^{29}$ Nova Zelândia ${ }^{30}$, Portugal, ${ }^{31}$ Canadá ${ }^{32}$ e Malásia. ${ }^{33}$ No Brasil, pesquisadores do Departamento de Engenharia Sanitária e Ambiental da Escola de Engenharia da UFMG iniciaram a investigação de águas residuárias de Belo Horizonte e Contagem (MG) com objetivo de identificar 
fragmentos virais de SARS-CoV-2, complementar estimativas oficiais obtidas por meio de testes clínicos e auxiliar no entendimento da circulação do novo coronavírus nas regiões investigadas. ${ }^{34} \mathrm{O}$ projeto-piloto tem sido conduzido pelo INCT ETEs Sustentáveis com apoio da Agência Nacional de Águas (ANA) e do Instituto Mineiro de Gestão das Águas (Igam).

Pesquisadores da Fiocruz, em parceria com a prefeitura de Niterói (RJ), também iniciaram estudo para verificar a presença de material genético do Sars-CoV-2 em amostras de esgoto bruto de doze pontos georreferenciados, em ETEs, na rede coletora de esgotos e em pontos de descarte de efluente hospitalar. Resultados iniciais positivos foram encontrados em cinco dos doze pontos amostrais. ${ }^{35}$ Finalmente, a CAESB e a Universidade de Brasília também estão trabalhando em ações conjuntas para identificar e quantificar o novo coronavírus nos esgotos afluentes de diferentes ETE do DF. ${ }^{36}$

\section{Estratégia para análise de SARS-CoV-2 e outros indicadores no esgoto}

A amostragem é uma das etapas mais importantes em investigações envolvendo a WBE. Isso ocorre devido a variações no aporte dos biomarcadores de interesse na rede de esgotamento sanitário, bem como por variações na vazão do esgoto que aflui na ETE ou em qualquer outro ponto amostral de interesse. Assim, amostras compostas são obtidas via alíquotas coletadas em intervalos pré-definidos ou, preferencialmente, em função da vazão do afluente. Neste caso, amostradores automáticos refrigerados, equipados com sistemas peristálticos e diversos sensores opcionais, têm sido usados para obtenção de amostras representativas de um período de $24 \mathrm{~h} .{ }^{37}$

Em vários países, o monitoramento de drogas de abuso via WBE tem sido realizado por meio da coleta de amostras em dias consecutivos por, pelo menos, uma semana. Estes trabalhos buscam avaliar o perfil semanal de consumo e associá-los ao estilo de vida nas sociedades urbanas. ${ }^{1,9}$ No caso do monitoramento de doenças infeciosas, como a COVID-19, os trabalhos até então publicados basearam-se em amostragens representativas ( $24 \mathrm{~h})$ com intervalos mais espaçados. É importante pontuar que, neste caso, o objetivo é monitorar curvas de contágio que podem se estender por semanas, meses ou mesmo anos. Neste caso, amostragens mais espaçadas, realizadas a cada semana, por exemplo, poderiam ser adotadas como forma de otimizar recursos financeiros e logísticos essenciais à realização das análises, principalmente em casos de doenças infecciosas que persistem por um tempo maior.

Durante um surto epidêmico, um determinado número de ETEs ou de pontos amostrais estratégicos, como elevatórias de esgoto, podem ser constantemente monitorados como forma de criar um mapa da dispersão de vírus. Com o esperado arrefecimento da curva de contágio, o número de ETEs ou pontos amostrais pode ser diminuído, sendo que em situações de controle, esgotos afluentes de somente uma ou duas ETEs podem continuar a serem monitorados, servindo como sentinelas para identificação de novos surtos. Neste caso, um sistema de alerta rápido poderia ser acionado com a consequente retomada da amostragem nos demais pontos amostrais.

A identificação e a quantificação de cargas virais do SARS-CoV-2 em amostras de esgoto têm se baseado na presença e quantificação de genes virais marcadores utilizando primers específicos por qRT-PCR e, eventualmente, na confirmação por técnicas de sequenciamento genético. ${ }^{24,25,28}$ Resumidamente, as amostras compostas de esgotos podem ser submetidas a um pré-tratamento térmico para a inativação de eventuais cargas virais remanescentes ${ }^{25}$ Em seguida, uma alíquota da amostra é retirada e submetida a diferentes etapas de pré-tratamento, como centrifugação ou filtração, para retirada de partículas em suspenção e células bacterianas. As etapas seguintes consistem na concentração das partículas virais (por centrifugação ou filtração) e extração do RNA viral com o auxílio de diferentes kits comercialmente disponíveis. ${ }^{24,29}$ Os extratos obtidos são então analisados por RT-qPCR para identificação de fragmentos de DNA amplificados pelos primers associados ao SARS-CoV-2 em equipamentos adequados. Análises quantitativas e de controle positivo têm sido realizadas via construção de curvas analíticas com plasmídeos contendo os genes marcadores disponíveis (Tabela 1). Para a confirmação de amostras positivas, os fragmentos amplificados na RT-qPCR podem ser sequenciados e comparados às sequências disponíveis em banco de dados públicos para SARS-CoV-2.

Alguns testes ainda podem demandar mais cuidado em termos de sensibilidade e especificidade, sendo que amostras de águas residuárias podem ser regularmente coletadas e congeladas para validação futura de métodos e reconstrução de tendências temporais da infecção. ${ }^{38}$ Portanto, esta estratégia pode ser usada para início imediato de amostragens em várias regiões do Brasil e do mundo de modo a prover, em futuro próximo, um banco de dados para melhor entendimento de surtos pandêmicos.

Tabela 1. Alguns primers (oligonucleotídeos iniciadores) de genes marcadores do vírus SARS-CoV-2.

\begin{tabular}{|c|c|c|c|}
\hline Gene Alvo & Primer & Sequência & Referência \\
\hline \multirow{2}{*}{ Glicoproteína (S) } & S-RPA-Forward_v1 & 5'-GAAATTAATACGACTCACTATAGGGAGGTTTCAAACTTTACTTGCTTTACATAGA-3' & 39 \\
\hline & S-RPA-Reverse_v1 & 5'-TCCTAGGTTGAAGATAACCCACATAATAAG-3' & 39 \\
\hline \multirow{2}{*}{ Envelope (E) } & E_Sarbeco_F & 5'-ACAGGTACGTTAATAGTTAATAGCGT-3' & 40 \\
\hline & E_Sarbeco_R & 5'-ATATTGCAGCAGTACGCACACA-3' & 40 \\
\hline \multirow{2}{*}{ Nucleocapsídeo (N) } & N_Sarbeco_F & 5'-CACATTGGCACCCGCAATC-3' & 40 \\
\hline & N_Sarbeco_R & 5'-GAGGAACGAGAAGAGGCTTG-3' & 40 \\
\hline \multirow{2}{*}{ Nucleocapsídeo (N1) } & 2019-nCoV_N1-F & 5'-GACCCCAAAATCAGCGAAAT-3' & 41 \\
\hline & 2019-nCoV_N1-R & 5'-TCTGGTTACTGCCAGTTGAATCTG-3' & 41 \\
\hline \multirow{2}{*}{ Nucleocapsídeo (N2) } & 2019-nCoV_N2-F & 5'-TTACAAACATTGGCCGCAAA-3' & 41 \\
\hline & 2019-nCoV_N2-R & 5'-GCGCGACATTCCGAAGAA-3' & 41 \\
\hline \multirow{2}{*}{ Nucleocapsídeo (N3) } & 2019-nCoV_N3-F & 5'-GGGAGCCTTGAATACACCAAAA-3' & 41 \\
\hline & 2019-nCoV_N3-R & 5'-TGTAGCACGATTGCAGCATTG-3' & 41 \\
\hline \multirow{2}{*}{$\mathrm{RdRp}$} & RdRp_SARSr-F & 5'-GTGARATGGTCATGTGTGGCGG-3' & 40 \\
\hline & RdRp_SARSr-R & 5'-CARATGTTAAASACACTATTAGCATA-3' & 40 \\
\hline
\end{tabular}




\section{Perspectivas e desafios}

Nos próximos meses, inúmeros resultados serão obtidos e publicados abordando o monitoramento da evolução de doenças infecciosas, como a COVID-19, em ambientes urbanos via análise de esgotos. Todos estes estudos devem consolidar ainda mais a WBE como uma estratégia alternativa, rápida e inovadora para estimar a exposição de comunidades e populações a substâncias químicas e patógenos. A criação de grupos multidisciplinares voltados ao monitoramento, entendimento e controle de epidemias deverá orientar a implementação da WBE para acompanhar surtos epidêmicos de outros vírus e agentes patógenos, como H1N1, rotavírus, adenovírus e norovírus via análises por qRT-PCR e estratégias aliadas como metagenoma e metatranscriptoma.

Modelagens computacionais sugerem que a WBE, aliada às técnicas tradicionais de vigilância, pode reduzir significativamente os custos necessários ao acompanhamento do SARS-CoV-2 em populações, economizando bilhões de dólares. ${ }^{42}$ Esta combinação seria mais crítica para as regiões mais pobres do mundo, onde testes laboratoriais e individuais para confirmação do contágio são escassos. Neste caso, a WBE poderia ser uma ferramenta disponível para orientar autoridades de saúde para tomada das ações necessárias. De fato, experiencias recentes no monitoramento global de genes indicadores de resistência antimicrobiana confirmam que a diversidade e a abundância de biomarcadores variam de acordo com a região e com as condições de saneamento, sendo que a vigilância de águas residuárias, especialmente em áreas com escassez de dados, pode ser particularmente informativa. ${ }^{43}$

A WBE pode contribuir para identificar curvas de contágio, comunidades infectadas e contribuições de assintomáticos, de modo a fomentar sistemas de alerta rápido para a presença de cargas virais. Em termos de vigilância em saúde, os resultados poderão subsidiar mapas de contágio e a consequente mobilização de recursos e profissionais para regiões prioritárias de maneira mais rápida e efetiva. Além disso, campanhas de conscientização e ações associadas poderão ser realizadas em cada comunidade, seja via mídias sociais e audiovisuais, bem como de maneira presencial em ambientes escolares, comerciais e hospitalares, por exemplo. Ainda, em futuro próximo, a WBE pode ser empregada para verificar a efetividade de vacinas para controle da COVID-19 em escala comunitária.

São muitos os argumentos que justificam a aplicação da WBE em um país como o Brasil, mais particularmente para o monitoramento imediato da epidemia do novo coronavírus. Contudo, deve-se levar em conta as particularidades brasileiras no desenvolvimento de estudos desta natureza, uma vez que são notórias as assimetrias nacionais em saneamento básico, tanto em termos geográficos quanto sociais, dificultando uma abordagem ampla da WBE. Tampouco serão obtidos resultados populacionais significativos de uma hora para outra, uma vez que demandam uma atuação técnico-científica permanente e em rede, agregando gestores públicos das áreas de saúde e saneamento a grupos de pesquisa diversos, em universidades ou outros centros. Somente países que se propõe a investir constantemente em pesquisa, desenvolvimento de recursos humanos em ciências e em instituições de referência serão capazes de tirar resultados do esgoto que "valem ouro", literalmente.

\section{AGRADECIMENTOS}

No Distrito Federal, os trabalhos envolvendo a epidemiologia do esgoto têm sido apoiados pela Companhia de Saneamento Ambiental do Distrito Federal (CAESB), pelo Conselho Nacional de Desenvolvimento Cientifico e Tecnológico (CNPq - Processo 429858/2018-3) e pela Fundação de Amparo à Pesquisa do Distrito Federal (FAPDF - Processo 19300000121/2019-13). Em âmbito nacional, os trabalhos contam com o apoio do Instituto Nacional de Ciências e Tecnologias Analíticas Avançadas (INCTAA) via Processos CNPq 573894/2008-6 e 465768/2014-8.

\section{REFERÊNCIAS}

1. Lorenzo, M.; Picó, Y.; Curr. Opin. Environ. Sci. Heal. 2019, 9, 77.

2. Choi, P. M.; Tscharke, B. J.; Donner, E.; O'Brien, J. W.; Grant, S. C.; Kaserzon, S. L.; Mackie, R.; O'Malley, E.; Crosbie, N. D.; Thomas, K. V.; Mueller, J. F.; TrAC - Trends Anal. Chem. 2018, 105, 453.

3. Castiglioni, S.; Borsotti, A.; Riva, F.; Zuccato, E.; Drug Alcohol Rev. 2016, 35, 128; Krizman, I.; Senta, I.; Ahel, M.; Terzic, S.; Sci. Total Environ. 2016, 566, 454; Lai, F. Y.; O’Brien, J.; Bruno, R.; Hall, W.; Prichard, J.; Kirkbride, P.; Gartner, C.; Thai, P.; Carter, S.; Lloyd, B.; Burns, L.; Mueller, J.; Sci. Total Environ. 2016, 568, 810; Castiglioni, S.; Borsotti, A.; Riva, F.; Zuccato, E.; Drug Alcohol Rev. 2016, 35, 128; Devault, D. A.; Maguet, H.; Merle, S.; Péné-Annette, A.; Lévi, Y.; Environ. Sci. Pollut. Res. 2018, 25, 27819.

4. EMCDDA, European Drug Report 2019: Trends an Developments; Lisbon, 2019.

5. UNODC, World Drug Report 2019 Methodology Report; 2019.

6. Maldaner, A. O.; Schmidt, L. L.; Locatelli, M. A. F.; Jardim, W. F.; Sodré, F. F.; Almeida, F. V.; Pereira, C. E. B.; Silva, C. M.; J. Braz. Chem. Soc. 2012, 23, 861; Sodré, F. F.; Souza, G. B.; Feitosa, R. S.; Pereira, C. E. B.; Maldaner, A. O.; J. Braz. Chem. Soc. 2017, 28, 2146; Sodré, F. F.; Feitosa, R. S.; Jardim, W. F.; Maldaner, A. O.; J. Braz. Chem. Soc. 2018, 29, 2287; Maldaner, A. O.; Sodré, F. F.; Freire, D. de J. S.; da Silva, K. M.; Perícia Fed. 2018, 42, 22; da Silva, K. M.; Quintana, J. B.; González-Mariño, I.; Rodil, R.; Gallassi, A. D.; Arantes, L. C.; Sodré, F. F.; Int. J. Environ. Anal. Chem. 2018, 98, 1370; González-Mariño, I.; Estévez-Danta, A.; Rodil, R.; Da Silva, K. M.; Sodré, F. F.; Cela, R.; Quintana, J. B.; Drug Test. Anal. 2019, 11, 1018.

7. Choi, P. M.; Thomas, K. V; O’Brien, J. W.; Mueller, J. F. In A New Paradigm for Environmental Chemistry and Toxicology: From Concepts to Insights; Jiang, G.; Li, X., Eds.; Springer Singapore: Singapore, 2020; pp. 99-114

8. Lopardo, L.; Adams, D.; Cummins, A.; Kasprzyk-Hordern, B.; Water Res. 2018, 143, 117.

9. Choi, P. M.; Tscharke, B.; Samanipour, S.; Hall, W. D.; Gartner, C. E.; Mueller, J. F.; Thomas, K. V; O'Brien, J. W.; Proc. Natl. Acad. Sci. 2019, 116, 21864.

10. Rousis, N. I.; Gracia-Lor, E.; Zuccato, E.; Bade, R.; Baz-Lomba, J. A.; Castrignanò, E.; Causanilles, A.; Covaci, A.; de Voogt, P.; Hernàndez, F.; Kasprzyk-Hordern, B.; Kinyua, J.; McCall, A.-K.; Plósz, B. G.; Ramin, P.; Ryu, Y.; Thomas, K. V; van Nuijs, A.; Yang, Z.; Castiglioni, S.; Water Res. 2017, 121, 270.

11. Gracia-Lor, E.; Zuccato, E.; Hernández, F.; Castiglioni, S.; J. Hazard. Mater. 2020, 382, 121108.

12. Xagoraraki, I.; O’Brien, E. In Women in Water Quality: Investigations by Prominent Female Engineers; O'Bannon, D. J., Ed.; Springer International Publishing: Cham, 2020; pp. 75-97.

13. Sims, N.; Kasprzyk-Hordern, B.; Environ. Int. 2020, 139, 105689.

14. Daughton, C.; Sci. Total Environ. 2020, 138149.

15. Mallapaty, S.; Nature 2020, 580, 176.

16. Stephens, P. R. S.; Oliveira, M. B. S. C. de; Ribeiro, F. C.; Carneiro, L. A. D. In Conceitos e Métodos para a Formação de Profissionais em Laboratórios de Saúde - Volume 4; Molinaro, E. M.; Caputo, L. F. G.; Amendoeira, M. R. R., Eds.; Fundação Osvaldo Cruz: Rio de Janeiro, 2009; pp. 125-220.

17. Walls, A. C.; Park, Y.-J.; Tortorici, M. A.; Wall, A.; McGuire, A. T.; Veesler, D.; Cell 2020, 181, 281.

18. Yan, R.; Zhang, Y.; Li, Y.; Xia, L.; Guo, Y.; Zhou, Q.; Science (80-. ). 2020, $367,1444$. 
19. Kampf, G.; Todt, D.; Pfaender, S.; Steinmann, E.; J. Hosp. Infect. 2020, 104, 246.

20. Yeo, C.; Kaushal, S.; Yeo, D.; Lancet Gastroenterol. Hepatol. 2020, 5 , 335.

21. Wu, Y.; Guo, C.; Tang, L.; Hong, Z.; Zhou, J.; Dong, X.; Yin, H.; Xiao, Q.; Tang, Y.; Qu, X.; Kuang, L.; Fang, X.; Mishra, N.; Lu, J.; Shan, H.; Jiang, G.; Huang, X.; Lancet Gastroenterol. Hepatol. 2020, 5, 434.

22. WHO, Water, sanitation, hygiene and waste management for the COVID-19 virus; 2020.

23. Lodder, W.; de Roda Husman, A. M.; Lancet Gastroenterol. Hepatol. 2020, Correspondance.

24. Medema, G.; Heijnen, L.; Elsinga, G.; Italiaander, R.; Brouwer, A.; medRxiv 2020, 2020.03.29.20045880.

25. Wu, F.; Xiao, A.; Zhang, J.; Gu, X.; Lee, W. L.; Kauffman, K.; Hanage, W.; Matus, M.; Ghaeli, N.; Endo, N.; Duvallet, C.; Moniz, K.; Erickson, T.; Chai, P.; Thompson, J.; Alm, E.; medRxiv 2020, 2020.04.05.20051540.

26. https://groups.google.com/forum/\#!forum/virus-surveillance-wastewater, acessada em Abril 2020.

27. https://www.protocols.io/groups/2019ncov-wastewater-epidemiology, acessada em Abril 2020

28. Wurtzer, S.; Marechal, V.; Mouchel, J.-M.; Moulin, L.; medRxiv 2020, 2020.04.12.20062679.

29. Ahmed, W.; Angel, N.; Edson, J.; Bibby, K.; Bivins, A.; O'Brien, J. W.; Choi, P. M.; Kitajima, M.; Simpson, S. L.; Li, J.; Tscharke, B.; Verhagen, R.; Smith, W. J. M.; Zaugg, J.; Dierens, L.; Hugenholtz, P.; Thomas, K. V; Mueller, J. F.; Sci. Total Environ. 2020, 138764.

30. https://www.nzherald.co.nz/nz/news/article.cfm?c_ id=1\&objectid=12323167, acessada em Abril 2020 .

31. https://www.publico.pt/2020/04/20/ciencia/noticia/portugal-vai-analisaraguas-residuais-detectar-sarscov2-1913017, acessada em Abril 2020.

32. https://www.watercanada.net/wastewater-test-could-provide-earlywarning-of-covid-19, acessada em Abril 2020.

33. https://www.bernama.com/en/news.php?id=1834528, acessada em Abril 2020.

34. https://ufmg.br/comunicacao/noticias/grupo-da-ufmg-executa-projetopiloto-de-monitoramento-da-covid-19-no-esgoto, acessada em Abril 2020.

35. https://portal.fiocruz.br/noticia/fiocruz-divulga-estudo-sobre-presencado-novo-coronavirus-em-esgotos-sanitarios, acessada em Maio 2020.

36. https://www.metropoles.com/colunas-blogs/janela-indiscreta/caesbe-unb-vao-investigar-se-novo-coronavirus-chegou-ao-esgoto-do-df, acessada em Maio 2020
37. Feitosa, R. S.; Sodré, F. F.; Maldaner, A. O.; Quim. Nova 2013, 36, 291.

38. Murakami, M.; Hata, A.; Honda, R.; Watanabe, T.; Environ. Sci. Technol. 2020.

39. Zhang, F.; Abudayyeh, O. O.; Gootenberg, J. S.; Bioarchive 2020, v. 20200321

40. Corman, V. M.; Landt, O.; Kaiser, M.; Molenkamp, R.; Meijer, A.; Chu, D. K. W.; Bleicker, T.; Schneider, J.; Schmidt, M. L.; Mulders, D. G. J. C.; Haagmans, B. L.; Veer, B. Van Der; Den, S. Van; Wijsman, L.; Goderski, G.; Ellis, J.; Zambon, M.; Peiris, M.; Goossens, H.; Reusken, C.; Koopmans, M. P. G.; Drosten, C.; Euro Surveill. 2019, 25, 2000045.

41. CDC 2019-Novel Coronavirus (2019-nCoV) Real-time rRT-PCR Panel Primers and Probes; 2020.

42. Hart, O. E.; Halden, R. U.; Sci. Total Environ. 2020, 138875.

43. Hendriksen, R. S.; Munk, P.; Njage, P.; van Bunnik, B.; McNally, L.; Lukjancenko, O.; Röder, T.; Nieuwenhuijse, D.; Pedersen, S. K.; Kjeldgaard, J.; Kaas, R. S.; Clausen, P. T. L. C.; Vogt, J. K.; Leekitcharoenphon, P.; van de Schans, M. G. M.; Zuidema, T.; de Roda Husman, A. M.; Rasmussen, S.; Petersen, B.; Bego, A.; Rees, C.; Cassar, S.; Coventry, K.; Collignon, P.; Allerberger, F.; Rahube, T. O.; Oliveira, G.; Ivanov, I.; Vuthy, Y.; Sopheak, T.; Yost, C. K.; Ke, C.; Zheng, H.; Baisheng, L.; Jiao, X.; Donado-Godoy, P.; Coulibaly, K. J.; Jergović, M.; Hrenovic, J.; Karpíšková, R.; Villacis, J. E.; Legesse, M.; Eguale, T.; Heikinheimo, A.; Malania, L.; Nitsche, A.; Brinkmann, A.; Saba, C. K. S.; Kocsis, B.; Solymosi, N.; Thorsteinsdottir, T. R.; Hatha, A. M.; Alebouyeh, M.; Morris, D.; Cormican, M.; O’Connor, L.; Moran-Gilad, J.; Alba, P.; Battisti, A.; Shakenova, Z.; Kiiyukia, C.; Ng'eno, E.; Raka, L.; Avsejenko, J.; Bērziņ̌̌, A.; Bartkevics, V.; Penny, C.; Rajandas, H.; Parimannan, S.; Haber, M. V.; Pal, P.; Jeunen, G.-J.; Gemmell, N.; Fashae, K.; Holmstad, R.; Hasan, R.; Shakoor, S.; Rojas, M. L. Z.; Wasyl, D.; Bosevska, G.; Kochubovski, M.; Radu, C.; Gassama, A.; Radosavljevic, V.; Wuertz, S.; Zuniga-Montanez, R.; Tay, M. Y. F.; Gavačová, D.; Pastuchova, K.; Truska, P.; Trkov, M.; Esterhuyse, K.; Keddy, K.; Cerdà-Cuéllar, M.; Pathirage, S.; Norrgren, L.; Örn, S.; Larsson, D. G. J.; Heijden, T. Van der; Kumburu, H. H.; Sanneh, B.; Bidjada, P.; Njanpop-Lafourcade, B.-M.; Nikiema-Pessinaba, S. C.; Levent, B.; Meschke, J. S.; Beck, N. K.; Van, C. D.; Phuc, N. Do; Tran, D. M. N.; Kwenda, G.; Tabo, D.; Wester, A. L.; Cuadros-Orellana, S.; Amid, C.; Cochrane, G.; Sicheritz-Ponten, T.; Schmitt, H.; Alvarez, J. R. M.; Aidara-Kane, A.; Pamp, S. J.; Lund, O.; Hald, T.; Woolhouse, M.; Koopmans, M. P.; Vigre, H.; Petersen, T. N.; Aarestrup, F. M.; Nat. Commun. 2019, 10, 1124 